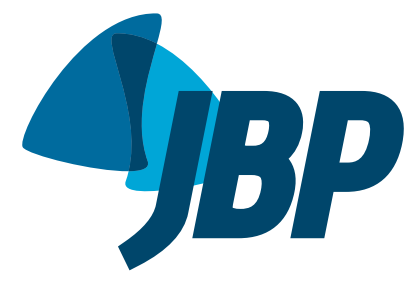

\title{
Usefulness of radiological signs of pulmonary congestion in predicting failed spontaneous breathing trials
}

\author{
Ana Carolina Peçanha Antonio',2, Cassiano Teixeira², Priscylla Souza Castro ${ }^{2,3}$, \\ Ana Paula Zanardo 2 , Marcelo Basso Gazzana², Marli Knorst ${ }^{4}$
}

1. Unidade de Terapia Intensiva Adulto, Hospital de Clínicas de Porto Alegre, Universidade Federal do Rio Grande do Sul, Porto Alegre (RS) Brasil.

2. Hospital Moinhos de Vento, Porto Alegre (RS) Brasil.

3. Unidade de Terapia Intensiva, Hospital Mãe de Deus, Porto Alegre (RS) Brasil.

4. Programa de Pós-Graduação em Pneumologia, Universidade Federal do Rio Grande do Sul, Porto Alegre (RS) Brasil.

Submitted: 6 December 2016.

Accepted: 18 June 2017

Study carried out at Hospital Moinhos de Vento, Porto Alegre (RS) Brasil.

\begin{abstract}
Objective: Inspiratory fall in intrathoracic pressure during a spontaneous breathing trial (SBT) may precipitate cardiac dysfunction and acute pulmonary edema. We aimed to determine the relationship between radiological signs of pulmonary congestion prior to an SBT and weaning outcomes. Methods: This was a post hoc analysis of a prospective cohort study involving patients in an adult medical-surgical ICU. All enrolled individuals met the eligibility criteria for liberation from mechanical ventilation. Tracheostomized subjects were excluded. The primary endpoint was SBT failure, defined as the inability to tolerate a T-piece trial for 30-120 min. An attending radiologist applied a radiological score on interpretation of digital chest X-rays performed before the SBT. Results: A total of 170 T-piece trials were carried out; SBT failure occurred in 28 trials (16.4\%), and 133 subjects (78.3\%) were extubated at first attempt. Radiological scores were similar between SBTfailure and SBT-success groups (median [interquartile range] $=3$ [2-4] points vs. 3 [24] points; $p=0.15)$, which, according to the score criteria, represented interstitial lung congestion. The analysis of ROC curves demonstrated poor accuracy (area under the curve $=0.58$ ) of chest $x$-rays findings of congestion prior to the SBT for discriminating between SBT failure and SBT success. No correlation was found between fluid balance in the $48 \mathrm{~h}$ preceding the SBT and radiological score results $(\rho=-0.13)$. Conclusions: Radiological findings of pulmonary congestion should not delay SBT indication, given that they did not predict weaning failure in the medical-surgical critically ill population.

(ClinicalTrials.gov identifier: NCT02022839 [http://www.clinicaltrials.gov/])

Keywords: Radiography; Pulmonary edema; Ventilator weaning.
\end{abstract}

\section{INTRODUCTION}

Weaning from mechanical ventilation (MV) is a gradual process that involves withdrawing the patient from the ventilator and removing the endotracheal tube. The weaning process can account for as much as $42 \%$ of the total duration of MV. ${ }^{(1-3)} \mathrm{MV}$ is associated with significant complications that are time-dependent in nature, prolonged intubation resulting in an increased incidence of complications, such as ventilator-associated pneumonia and increased mortality. ${ }^{(4,5)}$ However, impetuous attempts at weaning from MV are also hazardous. A failed attempt at extubation is associated with an 8-fold increase in the odds ratio for nosocomial pneumonia and a 6-to-12-fold increase in mortality risk. ${ }^{(6)}$ Therefore, the clinical challenge is to balance aggressiveness against safety.

Large clinical trials conducted in the 1990s showed that clinicians frequently miss opportunities to wean patients from MV. The fact that most patients (75\%) are extubated on the same day on which the weaning process is initiated suggests that the process can be initiated earlier, misconceptions and "slow" weaning procedures accounting for delayed weaning. $(7,8)$

Weaning-induced cardiac dysfunction is recognized as an important cause of weaning failure. ${ }^{(9)}$ During a spontaneous breathing trial (SBT), an abrupt drop in intrathoracic pressure during inhalation tends to increase the systemic venous return pressure gradient and to decrease the left ventricular (LV) ejection pressure gradient, thus resulting in increased LV filling pressure. A marked increase in the work of breathing can result in increased cardiac work and myocardial oxygen demand. ${ }^{(9,10)}$

Chest X-rays (CXRs) are commonly used in order to detect pulmonary edema. Radiographic signs that suggest accumulation of fluid in the lung interstitium or alveolar space include vascular redistribution, septal lines (Kerley's A and B lines), interlobular septal thickening, peribronchial cuffing, bilateral opacities ("bat wing" pattern), air bronchogram, and pleural effusion. In patients with pulmonary edema due to heart failure, the heart is commonly enlarged. ${ }^{(11,12)}$ Some experts recommend that CXRs be routinely taken before an SBT in order to confirm "disease reversal", discard fluid overload, and define eligibility. ${ }^{(2,13-15)}$ However, these criteria have been neither defined nor prospectively evaluated in a randomized controlled trial. In addition, CXR accuracy is significantly limited by acquisition techniques and clinical issues that override standardization procedures, especially in the ICU. ${ }^{(11,12)}$ 
Shochat et al. (16) developed a radiographic score (RS) to evaluate lung fluid content in individuals with acute heart failure following acute myocardial infarction. In that prospective single-center study, the novel RS was found to be able to assess lung edema severity and its changes over time, as well as correlating significantly with patient clinical status.

In a recent study, our research group showed loss of lung aeration during the weaning process, as estimated by bedside lung ultrasound; however, the presence of interstitial syndrome before initiation of weaning from MV failed to distinguish between individuals in whom weaning was successful and those in whom weaning failed. ${ }^{(17)}$ We assumed that CXR findings of lung edema also lack predictive power to discriminate between weaning success and weaning failure; therefore, radiological signs of pulmonary congestion should not delay the decision to initiate the weaning process. The objective of the present study was to assess prospectively whether radiological signs of pulmonary congestion prior to an SBT correlated with weaning outcomes in a heterogeneous group of mechanically ventilated patients.

\section{METHODS}

Between January of 2011 and March of 2013, nonconsecutive patients over 18 years of age and undergoing invasive MV for at least $24 \mathrm{~h}$ were selected from among those treated at a semiclosed medicalsurgical ICU in a private hospital, with 24-h intensivists. Patients with a tracheostomy were excluded. The local research ethics committee approved the study and waived the requirement for informed consent. The present study was a post hoc analysis of a prospective cohort study designed to investigate the potential role of lung ultrasound in predicting weaning outcomes.

Patients were assessed daily for eligibility to weaning according to the following parameters: clinical improvement of the underlying condition that led to acute respiratory failure; alertness and ability to communicate; adequate gas exchange, as indicated by $\mathrm{PaO}_{2}$ of at least $60 \mathrm{mmHg}$ and an $\mathrm{FiO}_{2}<0.40$; no significant respiratory acidosis (i.e., $\mathrm{pH}>7.3$ ); rapid shallow breathing index $\leq 105$ breaths/min/L; and vasoactive drugs at low and stable doses (norepinephrine doses $<0.12 \mu \mathrm{g} / \mathrm{kg} / \mathrm{min}$ or equivalent dopamine doses).

Attending physicians ordered digital CXRs on a heterogeneous pattern. Confirmation of disease resolution is a typical reason for the prescription of CXRs in our center, notably during MV. Anteroposterior CXR views were performed with the patients in the semi-upright position. For every ready-to-wean subject, a sole attending blinded radiologist was asked to interpret the most recent CXR available, usually obtained in the preceding 1-24 h, in accordance with the RS suggested by Shochat et al. (16) Then, the staff team, blinded to the CXR findings, coordinated ventilator discontinuation by means of a T-piece trial.

Each selected radiological sign of lung congestion was ascribed a predetermined value (Table 1 ), based on the sum of the RS sign scores: greater increases in lung fluid content represented higher RS scores, reflecting fluid accumulation. However, one single adjustment had to be made in one of the RS parameters: cardiothoracic ratio $\geq 60 \%$ was considered abnormal given that patients were in the semi-upright position during image acquisition. ${ }^{(18)}$ Examples of CXRs findings of lung edema are presented in Figures $1 \mathrm{~A}$ and $1 \mathrm{~B}$.

The primary outcome in this post hoc analysis was SBT failure, defined as the inability to tolerate a T-piece trial for 30-120 min, subjects not being extubated in this case. SBT was interrupted if the subject developed signs of respiratory discomfort (RR > 35 breaths/ min; arterial oxyhemoglobin saturation < $90 \%$; use of accessory respiratory muscles or paradoxical thoracoabdominal ventilation); tachycardia (HR > 140 bpm); hemodynamic instability (systolic blood pressure $<90 \mathrm{mmHg}$ or $20 \%$ over basal levels); or change in mental status (drowsiness, coma, or anxiety). There were no secondary endpoints in the present study.

Demographic data, including age, gender, and race, as well as comorbidities, severity of illness at the time of ICU admission, reason for MV initiation, physiological weaning predictors, and fluid balance (total inputs minus total outputs) $48 \mathrm{~h}$ before the SBT were recorded. The presence of diastolic or systolic LV dysfunction (the latter condition being defined as an ejection fraction $<45 \%$ ) was documented based on a formal echocardiogram report dated up to six months prior to admission. A diagnosis of COPD was based on history, physical examination, CXR, and previous pulmonary function tests, if available.

Shochat et al. ${ }^{(16)}$ found a mean raise of 4.8 points in the RS of individuals who developed overt acute heart failure during hospitalization, whose mean baseline values were 0.6 . Hence, our final sample of 170 subjects available for the analysis of the primary outcome had a $99 \%$ power to detect the same difference between SBT-success and SBT-failure groups, at a two-sided alpha level of 0.05 .

The results were expressed as mean $\pm \mathrm{SD}$, median (interquartile range $[\mathrm{IQR}]$ ), and proportions, as appropriate. The normal distribution of the various parameters was investigated by the distribution of data and using the Kolmogorov-Smirnov test. We used the Student's t-test or the Mann-Whitney $U$ test to compare continuous variables, and the chi-square test or Fisher's exact test to compare proportions, as appropriate. A ROC curve was generated based on predictive RS results and SBT outcomes. Spearman's correlation coefficient was determined in order to correlate fluid balance and RS results. The analyses were performed with the use of the IBM SPSS Statistics software package, version 20.0 (IBM Corporation, Armonk, NY, USA).

\section{RESULTS}

We obtained complete data for 170 weaning procedures. Overall, SBT failure occurred in 28 
(16.4\%). Table 2 shows the baseline characteristics of the cohort according to the outcome. Patients who were successfully extubated had been intubated for a shorter duration than had those who were not (median duration of MV: 4 days vs. 6 days; $p=0.003$ ). In our cohort, 133 patients (78.3\%) were extubated at first attempt. Sepsis of any source was the main reason for MV initiation, in approximately $40 \%$ of all individuals

Table 1. Radiological score parameters and values. ${ }^{\mathrm{a}}$

\begin{tabular}{ll}
\multicolumn{1}{c}{ Parameter } & Value \\
Redistribution of lung vessels & 0 \\
No & 1 \\
Yes & \\
Width of the cardiac silhouette $>60 \%$ & 0 \\
$\quad$ No & 1 \\
Yes & \\
Peribronchial cuffing & 0 \\
No & 1 \\
Yes & \\
New pleural effusion & 0 \\
No & 1 \\
Unilateral & 2 \\
Bilateral & \\
Kerley's A, B, or C lines & 0 \\
None & 1 \\
Uncertain & 2 \\
Definite & \\
Lung opacity & 0 \\
None & 1 \\
Lung opacity & 2 \\
Ground-glass opacity & \\
"Bat wing" pattern & 3 \\
\hline
\end{tabular}

Based on Shochat et al.(16) aSeverity of pulmonary edema was determined as follows: normal chest X-ray, 0-1 points; interstitial lung congestion, 2-4 points; and mild, moderate, and severe alveolar edema, respectively, 5-6 points, 7-8 points, and 9-10 points, respectively. in both groups. Approximately $11 \%$ of the individuals were intubated owing to congestive heart failure, and the same amount had a pre-existing diagnosis of systolic LV dysfunction.

The RS results were similar between SBT-failure and SBT-success groups (median $=3$ [IQR: 2-4] vs. 3 [IQR: 2-4]; $p=0.15$ ), which corresponded to interstitial lung congestion. The analysis of the ROC curve showed that the RS prior to a T-piece trial had poor accuracy in discriminating between SBT failure and SBT success (area under the curve $=0.58$; $p$ $=0.2$; Figure 2 ). There was no correlation between fluid balance $48 \mathrm{~h}$ prior to the SBT and RS results ( $\rho=$ $-0.13 ; p=0.1$ ).

\section{DISCUSSION}

In a heterogeneous cohort of mechanically ventilated patients who were candidates for a SBT, we found no association between radiological signs of pulmonary congestion indicated by the RS and SBT outcomes. Our study suggests that incorporating radiological estimations of lung edema as a readiness criterion for weaning from MV potentially retards it, as long as interstitial pulmonary congestion was observed on SBTs. To the extent of our knowledge, this is the first report encompassing such a topic.

The rationale behind delaying an SBT due to radiological signs of pulmonary congestion might be the belief that MV patients could not succeed in a T-piece trial unless they were "dry" again, given that cardiorespiratory interactions under negative pressure promote increases in both LV preload and afterload. However, a myriad of changes in respiratory mechanics and in the cardiovascular system related to weaning failure is not evident until clinical manifestations of distress appear, which promptly demand interruption of the trial or reintubation. ${ }^{(1)}$ Fluid balance cannot predict SBT outcomes in a slightly larger, mixed medical-surgical
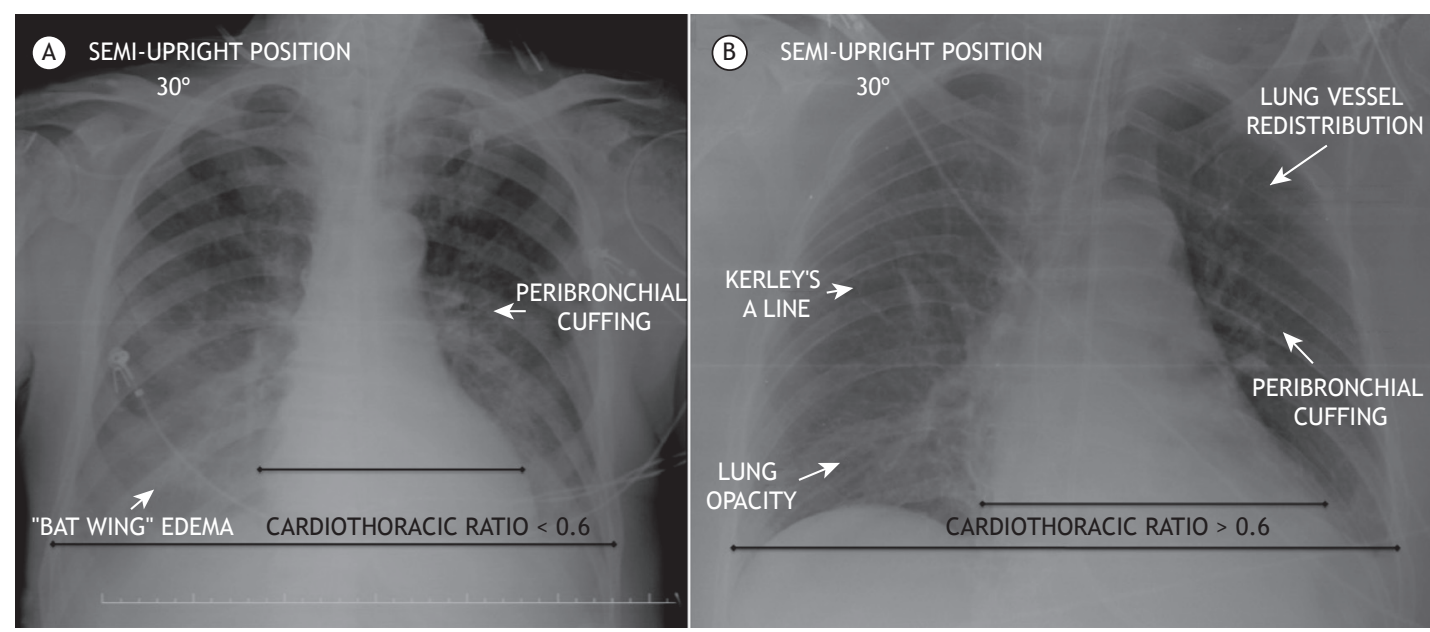

Figure 1. In A, a chest X-ray of a 68-year-old female patient shows peribronchial cuffing and opacity in a "bat wing" pattern, revealing edema, compounding a radiological score of 4 points, characterized as interstitial lung congestion.(16) In $B$, a chest X-ray of a 57 -year-old male patient shows a cardiothoracic ratio $>60 \%$, peribronchial cuffing, lung vessel redistribution, Kerley's A line, and lung opacity, resulting in a score of 5 points, characterized as mild alveolar edema. ${ }^{(16)}$ 
Table 2. Characteristics of the study cohort $(\mathrm{N}=170) .^{\text {a }}$

\begin{tabular}{|c|c|c|c|}
\hline \multirow[t]{2}{*}{ Characteristic } & \multicolumn{2}{|c|}{ Groups } & \multirow[t]{2}{*}{ p } \\
\hline & $\begin{array}{l}\text { SBT success } \\
(\mathrm{n}=142)\end{array}$ & $\begin{array}{l}\text { SBT failure } \\
(n=28)\end{array}$ & \\
\hline Age, years & $76(66-84)$ & $67(52-80)$ & 0.15 \\
\hline Female gender & $62(43.7)$ & $13(46.4)$ & 0.79 \\
\hline APACHE II score & $21 \pm 6.9$ & $23 \pm 7.8$ & 0.16 \\
\hline SOFA score & $5(3-9)$ & $5(2-10)$ & 0.50 \\
\hline $\mathrm{BMI}, \mathrm{kg} / \mathrm{m}^{2}$ & $25(23-28)$ & $25(23-29)$ & 0.97 \\
\hline$R S B I, f / V_{T}$ & $53(41-75)$ & $52(36-71)$ & 0.94 \\
\hline MV duration, days & $4(2-6)$ & $6(4-11)$ & 0.003 \\
\hline Fluid balance $48 \mathrm{~h}$ prior to the SBT, $\mathrm{mL}$ & $1,219 \pm 2,912$ & $1,838 \pm 1,896$ & 0.48 \\
\hline \multicolumn{4}{|l|}{ Comorbidities } \\
\hline COPD & $14(9.9)$ & $5(17.9)$ & 0.32 \\
\hline$E F<45 \%$ & $15(10.6)$ & $4(14.3)$ & 0.52 \\
\hline LV diastolic dysfunction & $55(38.7)$ & $8(28.6)$ & 0.30 \\
\hline Ischemic coronary disease & $28(19.7)$ & $4(14.3)$ & 0.50 \\
\hline RRT & $23(16.2)$ & $7(25.0)$ & 0.28 \\
\hline Presence of ascites & $3(2.1)$ & $2(7.1)$ & 0.19 \\
\hline \multicolumn{4}{|l|}{ Reason for MV } \\
\hline Respiratory sepsis & $25(17.6)$ & $6(21.4)$ & 0.63 \\
\hline Nonrespiratory sepsis & $32(22.5)$ & 5 (17.9) & 0.58 \\
\hline $\mathrm{CHF}$ & $18(12.7)$ & $1(3.6)$ & 0.16 \\
\hline Coma & $29(20.4)$ & $4(14.3)$ & 0.45 \\
\hline Postoperative ARF & $7(4.9)$ & $2(7.1)$ & 0.63 \\
\hline COPD/asthma & $2(1.4)$ & $2(7.1)$ & 0.13 \\
\hline Pulmonary embolism & $6(4.2)$ & $1(3.6)$ & 1.00 \\
\hline ARDS & $10(7.0)$ & $4(14.3)$ & 0.25 \\
\hline Simple weaning & $108(76.1)$ & $25(89.3)$ & 0.27 \\
\hline Vasopressor infusion during T-piece trial & $27(19.0)$ & $4(14.3)$ & 0.55 \\
\hline Vasodilator infusion during T-piece trial & $11(7.7)$ & $2(7.1)$ & 1.00 \\
\hline
\end{tabular}

SBT: spontaneous breathing trial; APACHE II: Acute Physiology and Chronic Health Evaluation II; SOFA: Sequential Organ Failure Assessment; BMI: body mass index; RSBI: rapid shallow breathing index; $f / \mathrm{V}_{\mathrm{T}}$ : ratio of RR to tidal volume; MV: mechanical ventilation; EF: ejection fraction; LV: left ventricular; RRT: renal replacement therapy; $\mathrm{CHF}$ : congestive heart failure; and ARF: acute respiratory failure. ${ }^{\text {aData }}$ are presented as median (interquartile range), mean $\pm \mathrm{SD}$, or $\mathrm{n}(\%)$.

ICU population either, perhaps being more relevant for COPD patients, as we published previously. ${ }^{(19)}$ An observational study involving 100 patients immediately before a T-piece trial demonstrated that baseline levels of brain natriuretic peptide-a surrogate marker of congestive heart failure-were moderately elevated exclusively in SBT-failure individuals, who eventually failed owing to cardiac dysfunction. (20) Moreover, overtreatment based on isolated CXR interpretations might be harmful in terms of weaning readiness. ${ }^{(10)}$

An interobserver agreement study examined the extent to which medical intensivists and a radiologist could agree on whether a CXR revealed diffuse bilateral infiltrates for the diagnosis of ARDS or not and concluded that intensivists without formal consensus training can achieve moderate levels of agreement. ${ }^{(21)}$ Accordingly, in real clinical practice, an expert radiological opinion is not immediately available, and delaying the weaning process based upon poor interpretations of CXRs might be even more questionable. Since the physicians in our study were not aware of the most recent CXR results, we were unable to prove that hypothesis. ${ }^{(24)}$
A systematic review ${ }^{(22)}$ highlighted the safety of abandoning routine CXRs in favor of a more restrictive approach. Arguments for adopting a restrictive approach included varying interpretations of CXRs depending on clinician and patient factors, low incidence of clinically unsuspected abnormalities, potential harm arising from unnecessary treatment of minor or false-positive findings, costs, radiation exposure and adverse events arising from the repositioning of the patient to obtain CXRs. (23) Likewise, the importance of negative CXR findings for workflow, efficiency, and clinical decisionmaking might be overestimated. A study collecting the opinions of experienced ICU physicians regarding the appropriateness of performing routine CXRs in various situations encountered in adult ICUs showed there was no consensus regarding the usefulness of obtaining a routine CXR prior to extubation. (24)

It should be pointed out that our study consisted of a relatively small number of patients and absolute number of failure events, with a high prevalence of elderly patients and a low prevalence of systolic LV dysfunction. Nonetheless, our sample had the same 

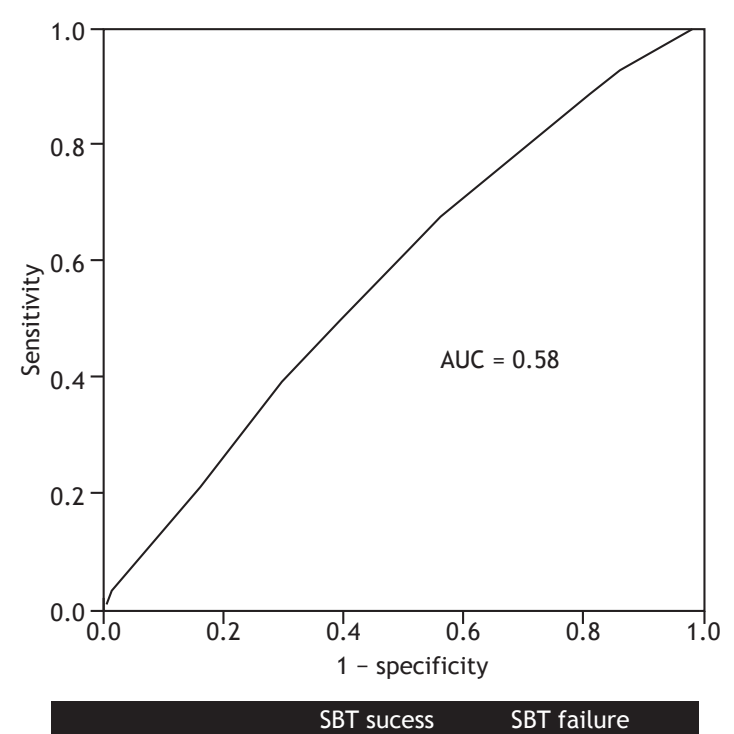

Radiological Score

$3(2-4)$

$3(2-4)$

Figure 2. A ROC curve of the ability of the radiological score to predict spontaneous breathing trial failure. The area under the curve (AUC) is $0.58(p=0.2)$, revealing poor accuracy.

expected pre-test probability of SBT failure as the ordinary medical-surgical ICU population. Our original study focused on lung ultrasound assessment of ready-to-wean subjects. Since CXR results were part of a secondary analysis, we did not standardize the moment of CXR acquisition, so not all exams were performed immediately before the SBT but rather within a period of $24 \mathrm{~h}$ prior to the trial. At the beginning of our research, the limited number of experts on bedside lung ultrasound, as well as lack of any research funding, forced us to close enrollment on weekends.

Other limitations include the observational design, with all its intrinsic methodological flaws, and the absence of high scores of radiological signs of lung edema on the RS, which might imply lesser overall severity in this cohort of patients or reflect the pointlessness in requiring CXRs to advance the weaning process. Our ready-to-wean population showed modest median values on the RS. In the original Shochat et al. paper, ${ }^{(16)}$ an RS of 4 or more represented overt acute heart failure in $95 \%$ of the patients reaching this level. Hence, it seems unlikely that an individual presenting with a high RS score should be eligible for an SBT.

We decided on SBT failure as the major outcome since we aimed at predicting the earliest time that a patient might resume spontaneous breathing. Moreover, the exact reason for extubation failure often escapes identification. Reintubation is usually performed because of an apparently new episode of respiratory distress, which may be related to primary respiratory failure, congestive heart failure, aspiration, ineffective cough causing accumulation of airway mucus, or upper airway obstruction. Other reasons for reintubation include the onset of a new episode of sepsis, surgical complications, acute coronary syndrome, and neurological impairment. ${ }^{(1)}$

The RS presented by Shochat et al. ${ }^{(16)}$ was chosen by virtue of its comprehensive analysis of dynamic changes, its good correlation with severity of lung edema, the utilization of lung impedance as the gold-standard method, and the sensitivity for the detection of subtle radiological signs of pulmonary congestion. Currently, that RS is the only method available that proposes a quantitative assessment of CXRs in terms of lung fluid content. In that cohort, ${ }^{(16)}$ high intraobserver ( $\mathrm{K}$ $=0.86 ; p=0.0001)$ and interobserver correlations ( $k=0.82 ; p=0.0001$ ) were found regarding RS interpretation. However, its main drawbacks are the lack of assessment in the ICU population-although it included patients admitted to a coronary care unit-its single-center design, and the absence of large-scale validation. Therefore, for the sake of generalizability of our findings, the RS must be further studied.

In conclusion, since the radiological signs of pulmonary congestion demonstrated by the RS were unable to predict SBT failure in this medical-surgical critically ill population, we inferred that specific CXR reports on signs of pulmonary congestion are insufficient to preclude hemodynamically stable, sufficiently oxygenated patients from performing an SBT.

\section{REFERENCES}

1. Tobin MJ. Weaning from Mechanical Ventilation. In: Tobin MJ, editor. Principles and Practice of Mechanical Ventilation. 3rd ed. New York: McGraw-Hill; 2012. p. 1185-220.

2. Maclntyre NR, Cook DJ, Ely EW Jr, Epstein SK, Fink JB, Heffner $J E$, et al. Evidence-based guidelines for weaning and discontinuing ventilatory support: a collective task force facilitated by the American College of Chest Physicians; the American Association for Respiratory Care; and the American College of Critical Care Medicine. Chest. 2001;120(6 Suppl):375S-95S. https://doi.org/10.1378/chest.120.6_ suppl.375S

3. Boles JM, Bion J, Connors A, Herridge M, Marsh B, Melot C, et al. Weaning from mechanical ventilation. Eur Respir J. 2007;29(5):103356. https://doi.org/10.1183/09031936.00010206

4. Cook DJ, Walter SD, Cook RJ, Griffith LE, Guyatt GH, Leasa D, et al. Incidence of and risk factors for ventilator-associated pneumonia in critically ill patients. Ann Intern Med. 1998;129(6):433-40. https://doi. org/10.7326/0003-4819-129-6-199809150-00002

5. Ely EW, Baker AM, Dunagan DP, Burke HL, Smith AC, Kelly PT, et al. Effect on the duration of mechanical ventilation of

identifying patients capable of breathing spontaneously. $\mathrm{N}$ Engl J Med. 1996;335(25):1864-9. https://doi.org/10.1056/ NEJM199612193352502

6. Frutos-Vivar F, Esteban A, Apezteguia C, González M, Arabi Y, Restrepo MI, et al. Outcome of reintubated patients after scheduled extubation. J Crit Care. 2011;26(5):502-9. https://doi.org/10.1016/j jcrc.2010.12.015

7. Esteban A, Frutos F, Tobin MJ, Alía I, Solsona JF, Valverdú I, et al. A comparison of four methods of weaning patients from mechanical ventilation. Spanish Lung Failure Collaborative Group. N Engl J Med. 1995;332(6):345-50. https://doi.org/10.1056/ NEJM199502093320601

8. Brochard L, Rauss A, Benito S, Conti G, Mancebo J, Rekik N, et al. Comparison of three methods of gradual withdrawal from ventilatory support during weaning from mechanical ventilation. Am J Respir Crit Care Med. 1994;150(4):896-903. https://doi.org/10.1164/ ajrccm.150.4.7921460

9. Teboul JL. Weaning-induced cardiac dysfunction: where are we today? Intensive Care Med. 2014;40(8):1069-79. https://doi 
org/10.1007/s00134-014-3334-4

10. Teboul JL, Monnet $\mathrm{X}$, Richard $\mathrm{C}$. Weaning failure of cardiac origin: recent advances. Crit Care. 2010;14(2):211. https://doi.org/10.1186/ cc8852

11. Lange NR, Schuster DP. The measurement of lung water. Crit Care. 1999;3(2):R19-R24. https://doi.org/10.1186/cc342

12. Khan AN, Al-Jahdali H, Al-Ghanem S, Gouda A. Reading chest radiographs in the critically ill (Part II): Radiography of lung pathologies common in the ICU patient. Ann Thorac Med. 2009;4(3):149-57. https://doi.org/10.4103/1817-1737.53349

13. Martin KT. Extubation: Guidelines and Procedures [monograph on the Internet]. [cited 2016 Nov 1] Corona, CA: Respiratory Care Educational Consulting Services Inc. and Western Schools. Available from: http://www.rcecs.com/MyCE/PDFDocs/course/N7020.pdf

14. Nickson C. Extubation Assessment in the ICU. Life in the Fastlane [serial on the Internet]. Mountain View, CA: Creative Commons; c2007-17 [cited 2015 Jul 9]; [about 10 screens]. Available from: http:// lifeinthefastlane.com/ccc/extubation-assessment/2014

15. Macintyre NR. Evidence-based assessments in the ventilator discontinuation process. Respir Care. 2012;57(10):1611-8. https:// doi.org/10.4187/respcare.02055

16. Shochat M, Shotan A, Trachtengerts V, Blondheim DS, Kazatsker $M$, Gurovich $V$, et al. A novel radiological score to assess lung fluid content during evolving acute heart failure in the course of acute myocardial infarction. Acute Card Care. 2011;13(2):81-6. https://doi. org/10.3109/17482941.2011.567279

17. Antonio AC, Teixeira C, Castro PS, Savi A, Maccari JG, Oliveira RP, et al. Behavior of Lung Ultrasound Findings during Spontaneous Breathing Trial. Rev Bras Ter Intensiva. Epub 2017.

18. van der Jagt EJ, Smits HJ. Cardiac size in the supine chestfilm. Eur J Radiol. 1992;14(3):173-7. https://doi.org/10.1016/0720048X(92)90080-S

19. Antonio AC, Teixeira C, Castro PS, Savi A, Oliveira RP, Gazzana $M B$, et al. 48-Hour Fluid Balance Does Not Predict a Successfu Spontaneous Breathing Trial. Respir Care. 2015;60(8):1091-6. https:// doi.org/10.4187/respcare.03172

20. Zapata L, Vera P, Roglan A, Gich I, Ordonez-Llanos J, Betbese AJ. B-type natriuretic peptides for prediction and diagnosis of weaning failure from cardiac origin. Intensive Care Med. 2011;37(3):477-85. https://doi.org/10.1007/s00134-010-2101-4

21. Meade MO, Cook RJ, Guyatt GH, Groll R, Kachura JR, Bedard $M$, et al. Interobserver variation in interpreting chest radiographs for the diagnosis of acute respiratory distress syndrome. Am $J$ Respir Crit Care Med. 2000;161(1):85-90. https://doi.org/10.1164/ airccm.161.1.9809003

22. Ganapathy A, Adhikari NK, Spiegelman J, Scales DC. Routine chest $x$-rays in intensive care units: a systematic review and meta-analysis. Crit Care. 2012;16(2):R68. https://doi.org/10.1186/cc11321

23. Tolsma $\mathrm{M}$, van der Voort $\mathrm{PH}$, van der Meer NJ. Why intensivists want chest radiographs. Crit Care. 2015;19:100. https://doi.org/10.1186/ s13054-015-0816-x

24. Hejblum G, loos V, Vibert JF, Böelle PY, Chalumeau-Lemoine L, Chouaid $C$, et al. A web-based Delphi study on the indications of chest radiographs for patients in ICUs. Chest. 2008:133(5):1107-12. https://doi.org/10.1378/chest.06-3014 